\section{Tratamiento estomatológico en el paciente adolescente con síndrome alcohólico fetal. Caso clínico}

\section{RESUMEN}

El síndrome alcohólico fetal es un conjunto de manifestaciones que se manifiestan en el recién nacido cuando la madre tomó bebidas alcohólicas durante el embarazo. Se presenta el caso clínico del tratamiento estomatológico de una adolescente de 17 años de edad con síndrome alcohólico fetal, con características intra- y extraorales, utilizando técnicas de manejo de conductas básicas y avanzadas con base en la clasificación de la American Academy of Pediatric Dentistry. Por otra parte, y con el objetivo de mejorar la calidad de vida de estos pacientes, se enfatiza la implementación de tratamientos estomatológicos del primer y segundo niveles de prevención, de acuerdo con la Organización Mundial de la Salud: promoción de la salud bucal, uso de selladores de fosetas y fisuras y la colocación de restauraciones de resina.

Palabras clave: síndrome alcohólico fetal, tratamiento estomatológico, paciente adolescente.

\section{Stomatological treatment in an adolescent affected by alcoholic fetal syndrome. Case report}

\begin{abstract}
Fetal alcohol syndrome is a set of events that occurs in newborns when the mother drinks alcohol during pregnancy. It is presenting a stomatological treatment of a teenager patient 17 year old with fetal alcohol syndrome with intra and extra oral features, using basic and advanced behavior techniques based on the classification of the American Academy of Pediatric Dentistry. Moreover, with the aim of improving the quality of life of these patients, the implementation of stomatological treatment with first and second level of prevention, according to WHO, as the promotion of oral health is emphasized, using pit and fissure sealants and placement of composite restorations.
\end{abstract}

Key words: Fetal alcohol syndrome, dental treatment, teenager patient.

\author{
Alicia Leonor Pinzón-Te ${ }^{1}$ \\ Rodrigo Serrano-Piña ${ }^{1}$ \\ Karen Ledezma-Gómez ${ }^{2}$ \\ ${ }^{1}$ Profesor de la Maestría en Odontología Infantil de \\ la Facultad de Odontología de la Universidad Autó- \\ noma de Yucatán. \\ ${ }^{2}$ Estudiante de la Licenciatura en Cirujano Dentista \\ en la Facultad de Odontología de la Universidad \\ Autónoma de Yucatán.
}

Recibido: 18 de enero del 2013

Aceptado: 16 de junio del 2014

Correspondencia: Dra. Alicia Leonor Pinzón Te Facultad de Odontología Universidad Autónoma de Yucatán

Calle 61-A No. 492

Col. Centro

CP 97000 Mérida, Yucatán

Tel: 9236752

Este artículo debe citarse como

Pinzón-Te AL, Serrano-Piña R, Ledezma-Gómez K. Tratamiento estomatológico en el paciente adolescente con síndrome alcohólico fetal. Caso clínico. Acta Pediatr Mex 2015;36:32-35. 


\section{INTRODUCCIÓN}

El síndrome alcohólico fetal se presenta en 30\% de las madres con alcoholismo. Es un conjunto de manifestaciones que se producen cuando las madres toman bebidas alcohólicas durante el embarazo por lo que el producto, al no contar con poder catalítico, queda indefenso.

Los principales signos y síntomas son hipocrecimiento prenatal y posnatal, escaso tejido adiposo, hipotonía, conducta hipopsíquica de aparición precoz o tardía, cardiopatía congénita, retraso mental, hiperactividad, déficit de atención, trastornos del lenguaje, mala coordinación, problemas de comportamiento de tipo cognitivo y psicosocial, microcefalia, hendiduras palpebrales de tamaño reducido, ptosis palpebral, hipoplasia maxilar, retrognatia en el lactante, micrognatia en el adolescente, labio superior fino, epicanto, miopía, estrabismo, hipoacusia, orejas prominentes, camptodactilia, aranodactilia del dedo índice, hipoplasia de uñas y luxación congénita de cadera. ${ }^{1-4}$ Las manifestaciones estomatológicas de este síndrome son enfermedad periodontal, caries, malas oclusiones, bruxismo, trastornos de articulación temporal mandibular, traumatismos, deglución atípica y respiración bucal.5,6

\section{CASO}

Adolecente de 17 años de edad que acudió a atención odontológica a la Clínica de Odontología Infantil, de la Facultad de Odontología de la Universidad Autónoma de Yucatán, por dolor al masticar. La paciente contaba con diagnóstico de síndrome alcohólico fetal: retraso mental por atrofia cerebral, mala coordinación motora, trastornos de lenguaje, hiperactividad, déficit de atención, reacciones impulsivas y ansiedad. Extraoralmente presentaba microcefalia, ptosis palpebral, hipoplasia maxilar, orejas prominentes, asimetría facial (Figuras 1 y 2). Intraoralmente

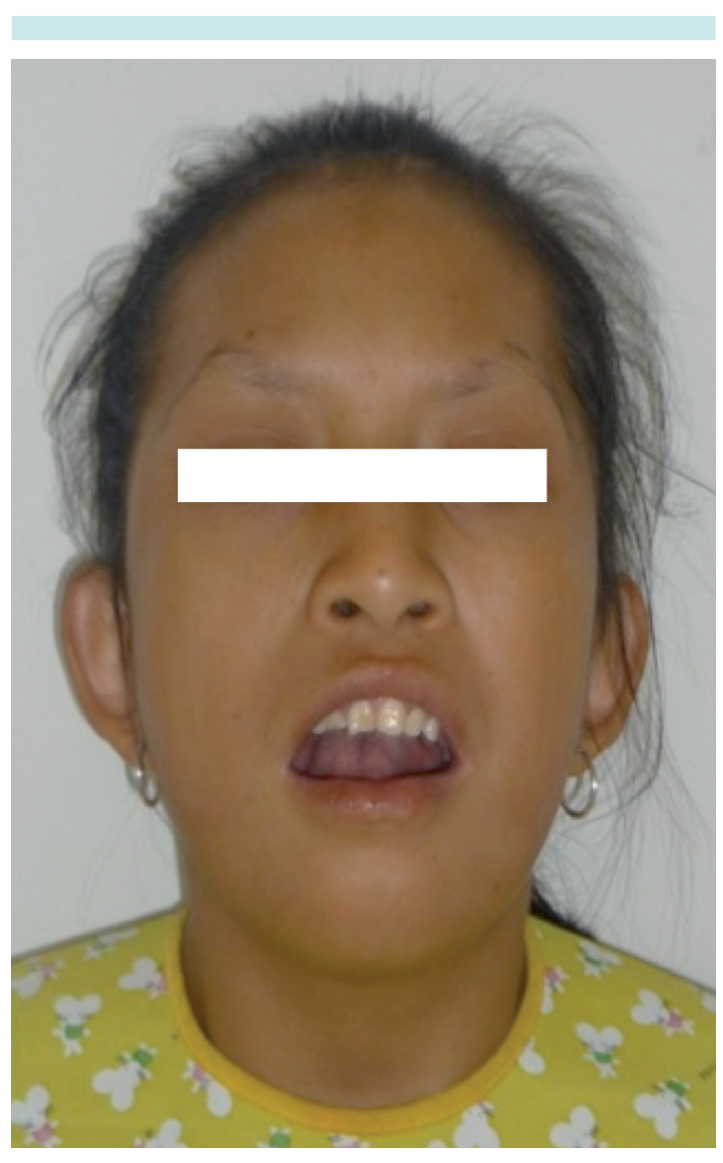

Figura 1. Características extraorales, vista frontal.

presentaba dentición permanente completa, apiñamiento dental, mala oclusión y múltiples caries (Figura 3). Actualmente está bajo tratamiento farmacológico con risperidona.

La conducta de la paciente se clasificó, con base en Frankl, como tipo I (definitivamente negativa) por lo que se decidió realizar el tratamiento estomatológico en el sillón dental de manera habitual (por motivos económicos), con el permiso de los padres plenamente informados. Se utilizaron técnicas de manejo de conducta: decir, mostrar y hacer, control de voz, ausencia de padres (técnicas básicas) e inmovilización por protección (técnica avanzada). 


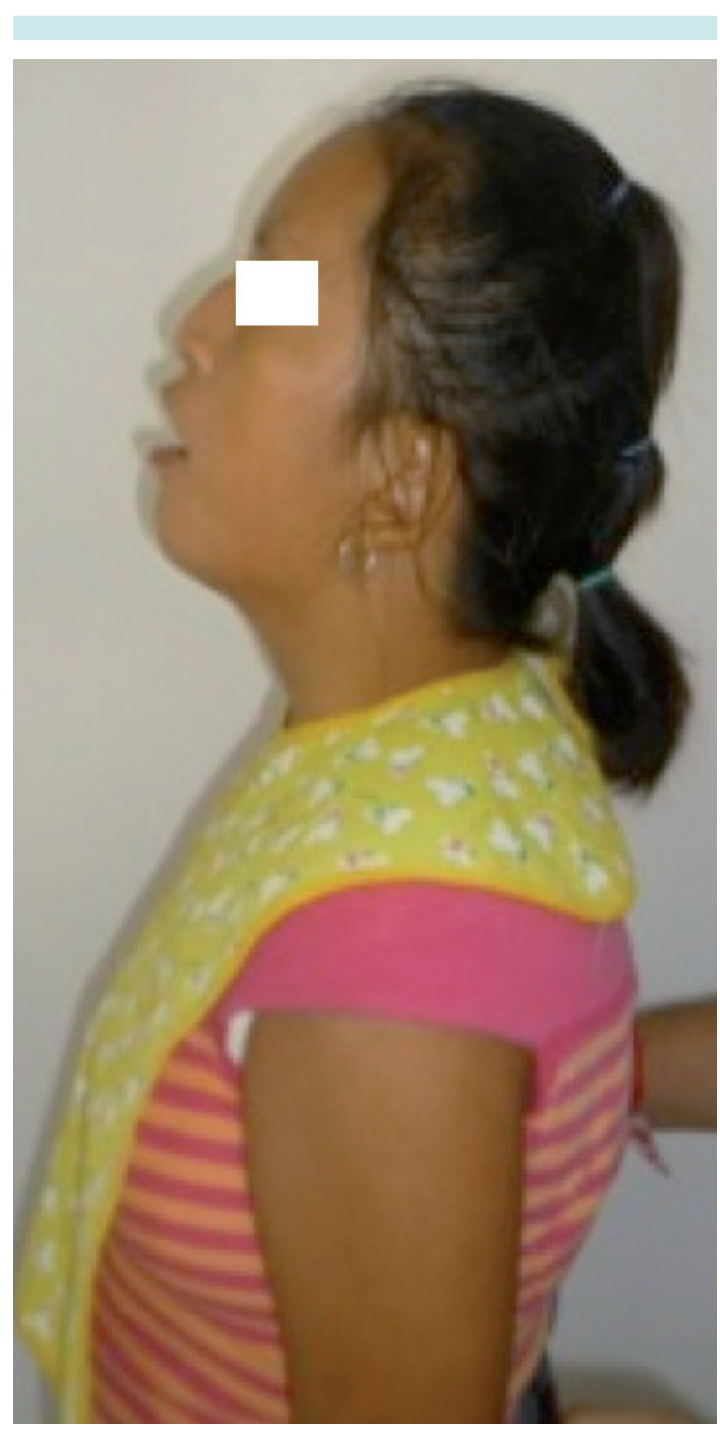

Figura 2. Características extraorales, vista lateral.

Se inició con la fase preventiva realizando control de la placa dentobacteriana, profilaxis de ambas arcadas y se instruyó a la madre de la paciente sobre la higiene bucal (primera cita); posteriormente se intervino por cuadrantes con aislamiento absoluto con dique de hule, para lo cual se colocaron selladores de fosetas y fisuras en premolares y resinas en molares (cuatro citas subsecuentes). En todas las citas se realizó el

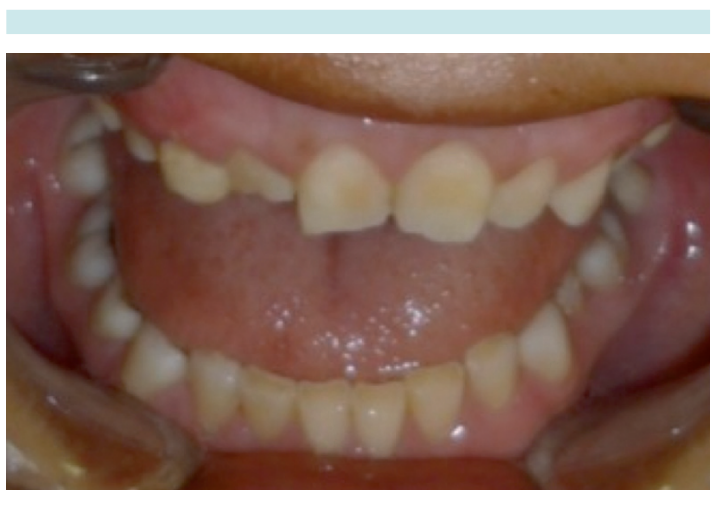

Figura 3. Características intraorales.

control de la placa dentobacteriana y se reforzó la técnica de cepillado para la promoción de la salud bucal. Cabe mencionar que al final del tratamiento la conducta de la paciente se clasificó como tipo III, ya que se observó mejoría durante las citas subsecuentes y sólo en las tres primeras citas fue necesaria la inmovilización por protección.

\section{Análisis}

Para el tratamiento integral de este tipo de pacientes es necesario un manejo multi- e interdisciplinario de neurólogo, rehabilitador físico, comunicación humana, psicólogo, ortopedista, cardiólogo y estomatólogo con el objetivo de producir una mejoría en la calidad de vida de estos pacientes. ${ }^{7}$ El estomatólogo debe conocer las características de este síndrome para proporcionar los tratamientos tanto preventivo como restaurativo pertinentes; éstos deben ir de la mano con el correcto manejo de la conducta del paciente durante la consulta. Así mismo, es importante recalcar que independientemente del nivel de prevención implementado para el tratamiento estomatológico, la promoción de la salud bucal sigue siendo el punto más importante en pacientes con este diagnóstico, con el objetivo de disminuir la necesidad de 
Pinzón-Te AL et al. Síndrome alcohólico fetal y tratamiento estomatológico

tratamientos restaurativos que muchas veces se dificultan por el costo y las alteraciones conductuales.

\section{CONCLUSIONES}

1. El síndrome alcohólico fetal requiere de atención multi- e interdisciplinaria.

2. Para realizar el tratamiento estomatológico el especialista deberá implementar las técnicas de manejo de conducta pertinentes en cada caso.

3. El tratamiento estomatológico preventivo en estos pacientes tiene como objetivo mejorar la calidad de vida y disminuir la necesidad de tratamientos restaurativos.

\section{REFERENCIAS}

1. Cruz M, Bosh J. Atlas de Síndromes Pediátricos. Edit. Espaxs, 1998. pp.321-322.

2. Varela CG, Chávez MM. Manifestaciones clínicas y odontológicas en el síndrome alcohólico fetal. Presentación de un caso. Archivos de Investigación materno infantil 2012;IV(2):79-85.

3. Cristóbal RC, Álvarez AN, Erazo K. Síndrome de Alcoholismo fetal. Reporte de un caso y Revisión de literatura. Honduras Pediátrica 2006;22(1):15-17.

4. Evrard SG. Criterios diagnósticos del síndrome alcohólico fetal y los trastornos del espectro del alcoholismo fetal. Archivos Argentinos de Pediatría 2010;108(1):61-67.

5. Fernández MD, Fernández JA. Fetopatía alcohólica: puesta al día. Revista de Neurología 2011;52(Supl 1):S53-7.

6. Sant'Anna LB, Tosello DO. Fetal alcohol syndrome and developing craniofacial and dental structures- a review. Orthod Craniofacial Res 2006;9:172-185.

7. Idrus NM, Thomas JD. Fetal alcohol spectrum disorders: experimental treatments and strategies for intervention. Alcohol Research \& Health 2011;34(1):76-85. 